\title{
Analysis of patients with tumor seeding after percutaneous radiofrequency ablation of hepatocellular carcinoma
}

\author{
KAZUE SHIOZAWA, MANABU WATANABE, NORITAKA WAKUI, TAKASHI IKEHARA, \\ KAZUNARI IIDA and YASUKIYO SUMINO

\begin{abstract}
Division of Gastroenterology and Hepatology, Toho University Medical Center, Omori Hospital, 6-11-1 Omorinishi, Ota-ku, Tokyo 143-8541, Japan
\end{abstract}

Received June 9, 2008; Accepted July 25, 2008

DOI: $10.3892 / \mathrm{mmr} 00000040$

\begin{abstract}
To determine the incidence and risk factors associated with tumor seeding after radiofrequency ablation (RFA) of hepatocellular carcinoma (HCC), 781 lesions from 352 patients who had undergone ultrasound (US)-guided RFA at our hospital between April 1999 and December 2005 were examined. Of these patients, 6 presented HCC lesions (6 lesions in total) and tumor seeding (7 seedings in total), which were analyzed. RFA using RITA 500 PA, the Cool-tip RFA System or the RTC 2000 System was performed. RFA sessions were repeated until complete necrosis was confirmed by imaging. Subsequently, follow-up was performed every 3-4 months by means of computed tomography (CT) and US scans. The 6 patients were retrospectively analyzed for patient characteristics, CT and histopathological findings, RFA method and complications, and clinical and imaging progress and outcome. Of the 6 lesions, 2 were in a subcapsular location at $\mathrm{S} 7$. Mean tumor diameter was $23.3 \pm 9 \mathrm{~mm}$. Tumor biopsies indicated that 1 of the 6 lesions was well-differentiated, 4 were moderately-differentiated, and 1 was undifferentiated. The RITA 500 PA was used in 2 cases, and the Cool-tip RFA System in 4. Seeding was identified $14.6 \pm 13$ months after RFA. Four of the cases with seeding were located on the abdominal wall, 2 on the thoracic wall and 1 in the Douglas pouch. Four of the patients underwent surgical resection, 1 radiation, and 1 conservative treatment for seeding. Five of the 6 patients died $12.6 \pm 9$ months after seeding was detected, with the exception having undergone surgical treatment. The seeding risks identified in this study include treatment of subcapsular lesions and patient treatment over multiple sessions. The selection of the proper RFA system to avoid multiple sessions and the use of ablation technique are important for the prevention of seeding. Additionally, long-term follow-up
\end{abstract}

Correspondence to: Dr Kazue Shiozawa, Division of Gastroenterology and Hepatology, Toho University Medical Center, Omori Hospital, 6-11-1 Omorinishi, Ota-ku, Tokyo 143-8541, Japan

E-mail:k.shio@med.toho-u.ac.jp

Key words: hepatocellular carcinoma, radiofrequency ablation, seeding, computed tomography, tumor biopsy after RFA by extensive imaging of the pelvic cavity and the thoracoabdominal wall is needed.

\section{Introduction}

Percutaneous treatments are effective for small non-surgical hepatocellular carcinoma (HCC). Recently, percutaneous radiofrequency ablation (RFA) therapy has emerged as an alternative to percutaneous ethanol injection therapy (PEIT) and percutaneous microwave coagulation therapy (PMCT). However, various studies have revealed complications associated with RFA, including a few studies on tumor seeding associated with the needle tract (1).

In general, the rates of tumor seeding that occur as a result of RFA for HCC are $0-2.8 \%$ (2-9), although one study reported a rate of $12.5 \%$ (1). The reported incidence varies from center to center as the mechanisms and factors involved in tumor seeding have not been identified, the number of patients analyzed has been small, the follow-up period short, and the methods of treatment varied. No studies have investigated tumor seeding and RFA methods using a sample group of more than 300 patients or long follow-up periods. In this study, we analyzed patients with tumor seeding after RFA for $\mathrm{HCC}$ in terms of patient characteristics and the development of tumor seeding complications following treatment.

\section{Materials and methods}

A total of 781 lesions from 352 patients who underwent ultrasound (US)-guided RFA instead of PEIT and PMCT as a local treatment for HCC in our hospital between April 1999 and December 2005 were examined. Of these patients, 6 ( 4 men and 2 women, age $66.5 \pm 12$ years) with $6 \mathrm{HCC}$ lesions and 7 tumor seedings (including 2 tumor seedings observed in 1 patient after 1 RFA session) were analyzed. Tumor seeding to the thoracoabdominal wall or pelvic cavity was confirmed by imaging during post-RFA follow-up.

The RFA systems used in the patients treated between April 1999 and May 2002 were the RITA 500 PA (RITA Medical System Inc., CA, USA) or the RTC 2000 System (Boston Scientific Inc., Natick, MA, USA), while in those treated between June 2002 and December 2005, the RTC 2000 System or the Cool-tip RFA System (Radionics Inc., Burlington, MA, USA) were used. 
RFA was performed using different needle electrodes as follows: i) the RITA 500 PA was used with RITA handpiece model 30, an expandable outer 14-gauge electrode. The electrode was fully extended, and initial power output was $20 \mathrm{~W}$ at $70^{\circ} \mathrm{C}$. After $3 \mathrm{~min}$, the temperature was set to $95-100^{\circ} \mathrm{C}$, and power output was increased to a maximum of $50 \mathrm{~W}$ and maintained for $>8$ min. ii) The Cool-tip RFA System was used with a Cool-tip 17-gauge single electrode. Initial power output was $40 \mathrm{~W}$. This was then increased by $10 \mathrm{~W}$ every minute to a maximum of $60 \mathrm{~W}$, and RF energy delivery was conducted three times until impedance increased beyond the limit of the generator. iii) The RTC 2000 System was used with a LeVeen expandable outer 15-gauge electrode. The electrode was slightly expanded at the base of the tumor according to its size, and initial power output was $30-40 \mathrm{~W}$. This was then increased by $10 \mathrm{~W}$ every minute until reaching the maximum suitable for the diameter of needle expansion, then maintained until roll-off occurred. Subsequently, the needle electrode was fully expanded and initial power output was set to $40 \mathrm{~W}$. This was increased by $10 \mathrm{~W}$ every minute until power output reached $90 \mathrm{~W}$. It was then maintained until roll-off occurred. After a 30-sec pause, power was reapplied at $70 \%$ of the maximum output achieved until power roll-off again occurred. If the area of necrosis was insufficient, the needle electrode was moved 5-10 mm upward under US monitoring, and ablation was repeated (stepwise expansion technique).

RFA sessions were repeated until complete necrosis was confirmed by imaging. Subsequently, follow-up was carried out every 3-4 months using computed tomography (CT) and US scans. The 6 patients were retrospectively analyzed for patient characteristics, CT and histopathological findings, RFA method and complications, and clinical and imaging progress and outcome (Table I).

\section{Results}

Of the 6 patients, 2 had type B, 3 type $C$ and 1 alcoholic cirrhosis, and 1 had ascites. Of the 6 lesions, 1 was located in $\mathrm{S} 3,1$ in S4 and 1 in S5, and 3 were in S7. Two of these 3 were in a subcapsular location. Mean tumor diameter was $23.3 \pm 9 \mathrm{~mm}$.

Contrast-enhanced CT revealed that all the lesions were enhanced in the early vascular phase. Tumor biopsies performed within the week before treatment showed that 1 of the 6 lesions was well-differentiated, 4 were moderatelydifferentiated and 1 was undifferentiated. In 4 of the lesions, one biopsy was conducted, while 2 biopsies were performed in the remaining 2. One of the 6 lesions was a local recurrence that arose following transcatheter arterial chemoembolization (TACE).

RFA was performed on 135 -mm-diameter lesion 1 month after TACE. The RITA 500 PA, the Cool-tip RFA System and the RTC 2000 System were used to ablate 2, 4 and 0 of the 6 lesions, respectively. The number of RFA sessions was 1 for 2 of the 6 lesions, 2 for 1, 3 for 1 , and 4 for 2 .

Treatment-associated complications, other than tumor seeding, were biloma in 2 patients, 1 of whom developed subcutaneous hematoma as well.

Tumor seeding was identified 14.6 \pm 13 months after RFA. The mean diameter of the seedings as shown by CT was 
A

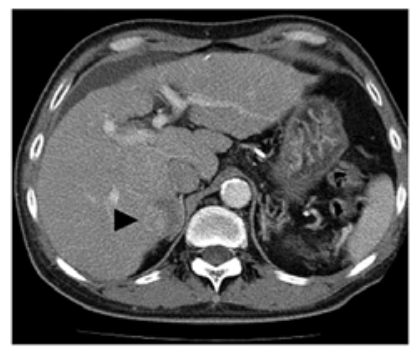

B

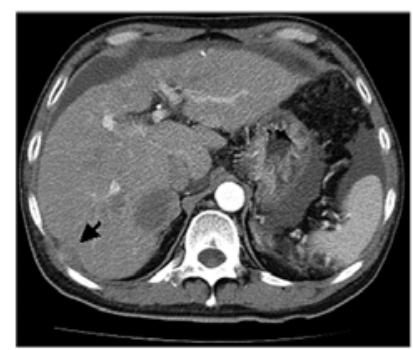

C

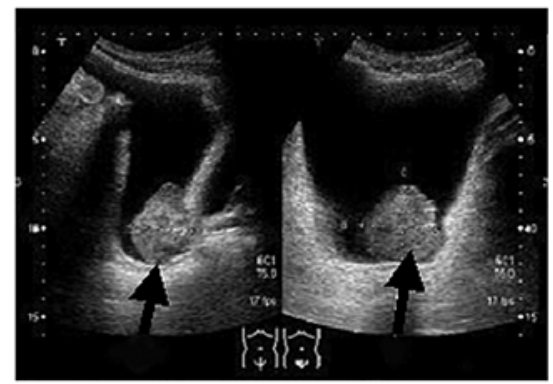

Figure 1. RFA using the Cool-tip RFA system with a Cool-tip electrode was performed for HCC in S7 with ascites (A) (arrowhead). Biloma occured after therapy. Four months after therapy, tumor seeding was detected on the abdominal wall (B) (arrow) and in the Douglas pouch (C) (arrow). Surgery was performed, and histopathology revealed the 2 tumor seedings to be moderately-differentiated HCC.

$20 \pm 8 \mathrm{~mm}$. All 7 lesions were of the nodular type. Four were located on the abdominal wall, 2 on the thoracic wall, and 1 in the Douglas pouch. In patients with ascites, tumor seeding was observed on the abdominal wall along the needle tract and in the Douglas pouch (Figs. 1 and 2).

Four patients underwent surgical resection, 1 radiation for tumor seeding, and 1 conservative treatment. Five of the 6 patients died of HCC $12.6 \pm 9$ months after the detection of seeding, with the exception having undergone surgical treatment. The characteristics of the 7 tumor seedings are listed in Table II.
A

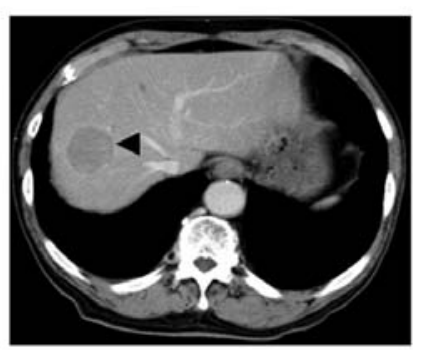

B

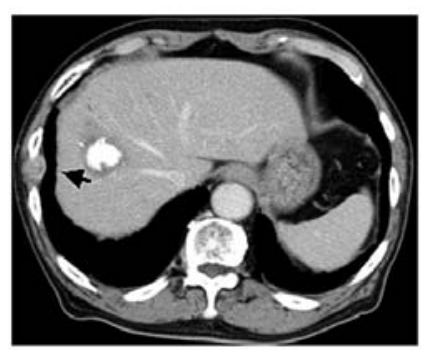

Figure 2. RFA using the Cool-tip RFA system with a Cool-tip electrode was performed for HCC in S7 1 month after TACE (A) (arrowhead). Thirteen months after the therapy, tumor seeding was detected on the right thoracic wall (B) (arrow). Surgery was performed.

\section{Discussion}

RFA was introduced in Japan in 1999 and is becoming increasingly established as standard therapy in various centers. It is less invasive than curative hepatectomy, yet has been reported to achieve comparable results in patients with tumors less than $30 \mathrm{~mm}$ in diameter (10). However, in recent years several studies have reported tumor seeding associated with the needle tract (1).

Several mechanisms may contribute to seeding. Viable tumor cells may adhere to the electrode during retraction. Tumor cells may also be carried into the tract with slight bleeding. Alternatively, cells may be forced into the tract by the sudden intratumoral hyperpressure frequently encountered during RFA, audible as a popping sound. Finally, cells may be driven in when saline is injected into the tumor during or before RFA $(3,11)$.

The rates of needle tract seeding of HCC after biopsy and after PEIT have been reported as $1.6-3.4 \%(12,13)$ and $1.1 \%$ (14), respectively. The rate of needle tract seeding of HCC after RFA is controversial as the reported incidence varies from center to center, with a range of $0-12.5 \%(1-9,15)$.

At our hospital, no tumor seeding complications occurred in patients who had undergone mainly PEIT for HCC prior to

Table II. Characteristics of the 7 tumor seedings.

\begin{tabular}{lcrrrr}
\hline Patient no. & Seeding size $(\mathrm{mm}) /$ location & \multicolumn{2}{c}{ Month detected (after RFA) } & Therapy for seeding $^{\text {D }}$ & Duration of survival $^{\mathrm{b}}$ \\
\hline 1 & 8/Thoracic wall & $7 / 2003$ & $(36)$ & Radiation & 27 \\
2 & 16/Abdominal wall & $2 / 2002$ & $(4)$ & No & 4 \\
3 & 23/Abdominal wall & $2 / 2004$ & $(11)$ & Surgery & 18 \\
4 & 28/Liver surface & $6 / 2004$ & $(4)$ & Surgery & 6 \\
& 32/Douglas pouch & $6 / 2004$ & $(4)$ & Surgery & Surgery \\
6 & 15/Abdominal wall & $7 / 2006$ & $(29)$ & Surgery & - \\
\hline
\end{tabular}

${ }^{a}$ The month/year when the tumor seeding was detected by CT or US (period in months from RFA until detection). ${ }^{b}$ Duration of survival (in months) after the detection of seeding. 
1999. In this study, in the 781 lesions treated with RFA between April 1999 and December 2005, the rate of tumor seeding was $0.7 \%$. In 2007, Stigliano et al (8) analyzed data from prior studies and reported the mean rate of tumor seeding to be $0.6 \%$, which is in approximate agreement with our results. The rates of tumor seeding in the present study by type of RFA system were $0.5 \%$ (2/386 lesions) for the RITA 500 PA and $1.7 \%$ (4/232 lesions) for the Cool-tip RFA System. The use of the RTC 2000 System on 163 lesions was not associated with tumor seeding.

Llovet et al (1) listed the following as factors that lead to tumor seeding: i) a direct, perpendicular approach to subcapsular tumors, ii) poor differentiation of the tumors, iii) patients with high AFP levels, and iv) the use of the Cool-tip RFA System.

In this study, subcapsular tumors accounted for 2 of the 6 lesions $(33.3 \%)$. Tumor biopsy showed that 5 of the lesions were moderately-differentiated or undifferentiated. AFP levels were not correlated with tumor seeding (data not shown).

Previous studies have reported that the use of a Cool-tip electrode is associated with a higher incidence of tumor seeding than the use of an expandable electrode $(1,16)$. Unlike its expandable counterpart, when using a Cool-tip more than 1 session is sometimes necessary to achieve a sufficient area of necrosis, due to its having only a single needle electrode. In this study, the mean number of sessions required with the RITA 500 PA was $1.5 \pm 0.7$, whereas with the Cool-tip it was 3 1.4. The RTC 2000 System calls for a stepwise expansion technique to be used. Consequently, a sufficient area of necrosis is achieved in a single session, thereby preventing the unnecessary extrusion of insufficiently coagulated cancer cells from the tumor.

As the mean diameter of HCC tumors in patients with

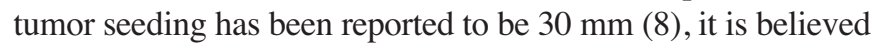
that the greater the tumor diameter, the higher the rate of tumor seeding. This is presumably because the tumor diameter reflects the poor differentiation of the tumor, and thus curative treatment requires a greater number of sessions. The mean diameter of HCC in the patients analyzed in this study was $23.3 \pm 9 \mathrm{~mm}$, with $2 \mathrm{HCC}$ lesions of more than $30 \mathrm{~mm}$ in diameter. We make a point of performing RFA combined with TACE for HCC tumors over $30 \mathrm{~mm}$ in diameter to reduce the cooling effect of neoplastic arterial blood flow, thereby achieving a sufficient area of necrosis. This has been performed in about $67 \%$ of patients. Of the patients who developed tumor seeding, 1 with HCC over $30 \mathrm{~mm}$ in diameter underwent RFA combined with TACE. The Cool-tip RFA System was used for this patient, and the number of sessions requried was 3 . This presumably caused the tumor seeding, and suggests that not only the combination of RFA with TACE, but also the selection of an RFA system suitable for the tumor diameter, is important in the case of large HCCs. The 7 tumor seedings appeared on the thoracoabdominal wall along the needle tract and exhibited enhancement similar to that of the primary lesion in the early vascular phase of contrast-enhanced CT. One lesion was observed in the Douglas pouch, necessitating follow-up by imaging including the pelvic cavity. Studies have reported that tumor seeding appeared approximately 7-13.6 months after RFA $(8,16)$. In this study, tumor seeding was detected within a year of RFA in 4 of the 6 patients, and at 28 and 36 months after RFA in some, suggesting the need for long-term follow-up after RFA therapy.

Based on our experiences, we have been applying the following treatment strategies since 2006: i) selection of an RFA system suitable for the location and size of the tumor to reduce the number of sessions, ii) a gradual rise in power output to prevent a rapid increase in intratumoral pressure, iii) adoption of the stepwise expansion technique with the RTC 2000 System, iv) cauterization of the needle tract, and v) the use of a US-guided needle.

Stigliano et al (8) reported that patients who had undergone a tumor biopsy before RFA therapy developed tumor seeding more frequently than those who had not. Therefore, the possibility that tumor biopsy itself causes tumor seeding cannot be excluded. However, we performed tumor biopsies as needed, both before and after 2006, and did not observe any resulting differences. The only difference between the two periods was the RFA strategy followed, including the selection of RFA system.

Between January 2006 and June 2007, 259 HCC lesions in 153 patients were treated with RFA using the Cool-tip RFA System for 168 lesions and the RTC Generator System for 91 nodules. As of February 2008, after 26 months, no seeding had been observed. No significant difference was noted in the treatment results due to differences in the RFA system in the time before and after 2006. Nonetheless, we believe that multiple sessions with the Cool-tip RFA System are an important factor affecting tumor seeding incidence. Currently, we adhere to a policy of selecting an RFA system suitable for the tumor diameter, and use the Cool-tip RFA System mainly for HCC lesions less than $20 \mathrm{~mm}$ in diameter. This achieves a sufficient area of necrosis in a single session in almost all patients. However, various other factors appear to be involved in tumor seeding and, since it can surface several years after RFA therapy, subsequent and attentive follow-up is needed.

Based on our retrospective study of $6 \mathrm{HCC}$ patients who developed tumor seeding after RFA, we conclude that seeding risks include poorly-differentiated tumors and multiple RFA sessions. The selection of an RFA system that reduces the number of sessions required, as well as the ablation technique used, are important for the prevention of tumor seeding. Additionally, long-term follow-up after RFA by extensive imaging of the pelvic cavity and the thoracoabdominal wall is needed.

\section{References}

1. Llovet JM, Vilana R, Bru C, Bianchi L, Salmeron JM, Boix L, Ganau S, Sala M, Pages M, Ayuso C, Sole M, Rodes J and Bruix J: Increased risk of tumor seeding after percutaneous radiofrequency ablation for single hepatocellular carcinoma. Hepatology 33: 1124-1129, 2001.

2. Livraghi T, Goldberg SN, Lazzaroni S, Meloni F, Sobiati L and Gazelle GS: Small hepatocellular carcinoma: treatment with radio-frequency ablation versus ethanol injection. Radiology 210 : 655-661, 1999.

3. Mulier S, Mulier P, Ni Y, Miao Y, Dupas B, Marchal G, Wever ID and Michel L: Complications of radiofrequency coagulation of liver tumors. Br J Surg 89: 1206-1222, 2002.

4. Livraghi T, Solbiati L, Meloni MF, Gazelle GS, Halpern EF and Goldberg SN: Treatment of focal liver tumors with percutaneous radio-frequency ablation: complications encountered in a multicenter study. Radiology 226: 441-451, 2003. 
5. De Baere T, Risse O, Kuoch V, Dromain C, Sengel C, Smayra T, Din MG, Letoublon $C$ and Elias D: Adverse events during radiofrequency treatment of 582 hepatic tumors. AJR Am J Roentgenol 181: 695-700, 2003.

6. Jskolka JD, Asch MR, Kachura JR, Ho CS, Ossip M, Wong F, Sherman M, Grant DR, Greig PD and Gallinger S: Needle tract seeding after radiofrequency ablation of hepatic tumors. J Vasc Interv Radiol 16: 485-491, 2005.

7. Chen MH, Wei Y, Yan K, Gao W, Dai Y, Huo L, Yin SS, Zhang H and Poon RTP: Treatment strategy to optimize radiofrequency ablation for liver malignancies. J Vasc Interv Radiol 17: 671-683, 2006.

8. Stigliano R, Marelli L, Yu D, Davies N, Patch D and Burroughs AK: Seeding following percutaneous diagnostic and therapeutic approaches for hepatocellular carcinoma. What is the risk and the outcome? Seeding risk for percutaneous approach of HCC. Cancer Treat Rev 33: 437-447, 2007.

9. Shirato K, Morimoto M, Tomita N, Kokawa A, Sugimori K, Saito T and Tanaka K: Hepatocellular carcinoma: a case of extrahepatic seeding after percutaneous radiofrequency ablation using an expandable needle electrode. Hepatogastroenterology 49: 897-899, 2002.

10. Poon RT, Fan ST, Tsang FH and Wong J: Locoregional therapies for hepatocellular carcinoma: a critical review from surgeon's perspective. Ann Surg 235: 466-486, 2002.
11. Miao Y, Ni Y, Mulier S, Wang K, Hoey MF, Mulier P Penninckx F, Yo J, De Scheerder I, Baert AL and Marchal G: Ex vivo experiment on radiofrequency liver ablation with saline infusion through a screw-tip cannulated electrode.J Surg Res 71: 19-24, 1997.

12. Durand F, Regimbeau JM, Belghiti J, Sauvanet A, Vilgrain V, Terris B, Moutardier V, Farges O and Valla D: Assessment of the benefits and risks of percutaneous biopsy before surgical resection of hepatocellular carcinoma. J Hepatol 35: 254-258, 2001.

13. Kim SH, Lim HK, Lee WJ, Cho JM and Jang HJ: Needle-tract implantation in hepatocellular carcinoma: frequency and CT findings after biopsy with the 19.5-gauge automated biopsy gun. Abdom Imaging 25: 246-250, 2000.

14. Ishii H, Okada S, Okusaka T, Yoshimori M, Nakasuka H, Shimada K, Yamasaki S, Nakanishi Y and Sakamoto M: Needle tract implantation of hepatocellular carcinoma after percutaneous ethanol injection. Cancer 82: 1638-1642, 1998.

15. Bolondi L, Gaiani S, Celli N and Piscaglia F: Tumor dissemination after radiofrequency ablation of hepatocellular carcinoma. Hepatology 34: 608, 2001.

16. Livraghi T, Lazzaroni S, Meloni F and Solbiati L: Risk of tumor seeding after percutaneous radiofrequency ablation for hepatocellular carcinoma. Br J Surg 92: 856-858,2005. 